\title{
SCALING LIMIT FOR A DRAINAGE NETWORK MODEL
}

\author{
C. F. COLETTI, ${ }^{* * *}$ \\ L. R. G. FONTES **** AND \\ E. S. DIAS, ${ }^{* * * *}$ Universidade de São Paulo
}

\begin{abstract}
We consider the two-dimensional version of a drainage network model introduced in Gangopadhyay, Roy and Sarkar (2004), and show that the appropriately rescaled family of its paths converges in distribution to the Brownian web. We do so by verifying the convergence criteria proposed in Fontes, Isopi, Newman and Ravishankar (2002).
\end{abstract}

Keywords: Drainage network; coalescing random walk; Brownian web; coalescing Brownian motion

2000 Mathematics Subject Classification: Primary 60K35; 60K40; 60F17

\section{Introduction and results}

\subsection{The two-dimensional drainage network model}

Let $\mathbb{Z}$ be the set of integers, and let $\Omega=\left(\omega(z), z \in \mathbb{Z}^{2}\right)$ be a family of Bernoulli independent random variables with parameter $p \in(0,1)$. Let $\mathrm{P}_{p}$ and $\mathrm{E}_{p}$ respectively be the probability and expectation induced by these variables in the product space $(\{0,1\})^{\mathbb{Z}^{2}}$. Consider a second family $\Upsilon=\left(v(z), z \in \mathbb{Z}^{2}\right)$ of Bernoulli independent random variables with parameter $\frac{1}{2}$. Let $\mathrm{P}_{1 / 2}$ and $\mathrm{E}_{1 / 2}$ be the probability and expectation induced by these variables in the product space $(\{0,1\})^{\mathbb{Z}^{2}}$. Let $\mathrm{P}=\mathrm{P}_{p} \times \mathrm{P}_{1 / 2}$ be the product probability induced by the Bernoulli variables in the product space $\{0,1\}^{\mathbb{Z}^{2}} \times\{0,1\}^{\mathbb{Z}^{2}}$, and let $\mathrm{E}$ be the expectation operator with respect to this probability.

For $z=\left(z_{1}, z_{2}\right) \in \mathbb{Z}^{2}$, we say that $z^{\prime}$ is in the next level if $z^{\prime}=\left(w, z_{2}+1\right)$ for some $w \in \mathbb{Z}$. Let $h(z)$ be the closest open vertex to $z$ in the next level with respect to the distance induced by the $l_{1}$-norm. That is to say, $\omega(h(z))=1$ and $\sum_{i=1}^{2}\left|h(z)_{i}-z_{i}\right|=\min \left\{\sum_{i=1}^{2}\left|x_{i}-z_{i}\right|: \omega(x)=1\right.$ and $\left.x_{2}=z_{2}+1\right\}$. If there exist two closest open vertices to $z$ in the next level, the connection will be to its left if $v(z)=0$ and to its right if $v(z)=1$. Now, let $h^{0}(z)=z$ and iteratively, for $n \geq 1, h^{n}(z)=h\left(h^{n-1}(z)\right)$.

Now let $g=(V, \mathcal{E})$ be the random directed graph with vertices $V=\mathbb{Z}^{2}$ and edges $\mathcal{E}=\left\{(u, h(u)): u \in \mathbb{Z}^{2}\right\}$. This model was proposed by Gangopadhyay et al. [9] and will be called the GRS model for short.

Received 11 July 2008; revision received 12 August 2009.

* Postal address: Instituto de Matemática e Estatística, Universidade de São Paulo, Rua do Matão 1010, Cidade Universitária, 05508-090, São Paulo, Brazil.

** Email address: cristian@ime.usp.br

Research supported by FAPESP grant 2006/54511-2.

*** Email address: 1renato@ime.usp.br

Research partially supported by CNPq grants 307978/2004-4 and 484351/2006-0, and FAPESP grant 2004/07276-2.

**** Email address: dias@ime.usp.br

Research supported by FAPESP grant 2004/13008-0. 


\subsection{Main result}

It follows from the construction that the GRS model may be seen as a set of continuous paths, as follows. For any $z=\left(z_{1}, z_{2}\right) \in \mathbb{Z}^{2}$, we define the path $X^{z}=\left\{\left(X^{z}(s), s\right), s \geq z_{2}\right\}$ in $\mathbb{R}^{2}$ as the linearly interpolated line composed by all edges $\left\{\left(h^{k}(z), h^{k+1}(z)\right): k \in \mathbb{N}\right\}$ of the model, with $X^{z}(k)=h^{k}(z), k \in \mathbb{N}$, where $h^{k}$ is the $k$ th composition of $h, h^{0}$ meaning the identity. Clearly, $X^{z}$ is a continuous path starting at time $z_{2}$. We let

$$
X:=\left\{X^{z}: z \in \mathbb{Z}^{2}\right\}
$$

which we also call the drainage network, and consider its diffusive rescaling

$$
\mathcal{X}_{\delta}:=\left\{\left(\delta x_{1}, \delta^{2} x_{2}\right) \in \mathbb{R}^{2}:\left(x_{1}, x_{2}\right) \in \mathcal{X}\right\}
$$

for $\delta \in(0,1]$. Our main result below shows that $\mathcal{X}_{\delta}$ converges in distribution to the Brownian web.

Several authors constructed random processes that formally correspond to coalescing onedimensional Brownian motions starting from every space-time point [2], [1], [8], [7], [13]. In [8], the Brownian web is characterized as a random variable taking values in a metric space whose points are compact sets of paths. Denote by $\left(\mathcal{H}, d_{\mathscr{H}}\right)$ the complete separable metric space where the Brownian web is defined. Denote also by $\mathcal{F}_{\mathcal{H}}$ the corresponding Borel $\sigma$-algebra generated by $d_{\mathscr{H}}$.

The closure in path space of the rescaled drainage network $\mathcal{X}_{\delta}$, also denoted by $\mathcal{X}_{\delta}$, is an $\left(\mathscr{H}, \mathcal{F}_{\mathscr{H}}\right)$-valued random variable.

Theorem 1. The rescaled drainage network $\mathcal{X}_{\delta}$ converges in distribution to the Brownian web as $\delta \rightarrow 0$.

In order to prove Theorem 1, we will verify the convergence criteria of [8]. To describe them, we need the following definition. Given $t_{0} \in \mathbb{R}, t>0, a<b$, and an $\left(\mathscr{H}, \mathcal{F}_{\mathcal{H}}\right)$-valued random variable $\mathcal{V}$, let $\eta_{\mathcal{\nu}}\left(t_{0}, t ; a, b\right)$ be the $\{0,1,2, \ldots, \infty\}$-valued random variable giving the number of distinct points in $\mathbb{R} \times\left\{t_{0}+t\right\}$ that are touched by paths in $\mathcal{V}$ which also touch some point in $[a, b] \times\left\{t_{0}\right\}$.

We can now state the convergence criteria. Let $\mathcal{D}$ be a countable dense set of points in $\mathbb{R}^{2}$.

Theorem 2. ([8].) Suppose that $\mathcal{X}_{1}, \mathcal{X}_{2}, \ldots$ are $\left(\mathcal{H}, \mathcal{F}_{\mathcal{H}}\right)$-valued random variables with noncrossing paths. If, in addition, the following three conditions are valid, the distribution of $\mathcal{X}_{n}$ converges to the distribution of the standard Brownian web.

(I1) There exist $\theta_{n}^{y} \in X_{n}$ such that, for any deterministic $y_{1}, \ldots, y_{m} \in \mathscr{D}, \theta_{n}^{y_{1}}, \ldots, \theta_{n}^{y_{m}}$ converge in distribution as $n \rightarrow \infty$ to coalescing Brownian motions (with unit diffusion constant) starting at $y_{1}, \ldots, y_{m}$.

(B1) $\lim \sup _{n \rightarrow \infty} \sup _{\left(a, t_{0}\right) \in \mathbb{R}^{2}} \mathrm{P}\left(\eta_{\chi_{n}}\left(t_{0}, t ; a, a+\varepsilon\right) \geq 2\right) \rightarrow 0$ as $\varepsilon \rightarrow 0+$.

(B2) $\varepsilon^{-1} \lim \sup _{n \rightarrow \infty} \sup _{\left(a, t_{0}\right) \in \mathbb{R}^{2}} \mathrm{P}\left(\eta_{\chi_{n}}\left(t_{0}, t ; a, a+\varepsilon\right) \geq 3\right) \rightarrow 0$ as $\varepsilon \rightarrow 0+$.

The present model can be viewed as a discrete space, long-range version of the twodimensional Poisson tree introduced in [6]. In [5], the weak convergence to the Brownian web of the rescaled collection of paths of that model was established. The approach here is the same as the one pursued there. There are considerable technical differences in the present work though. The main ones are as follows: (a) the interaction among the paths in the present 
model is a long-range interaction, while in the former case it is a local interaction; this requires considerably more care in the proofs of (I1) and of an estimate on the tail of the distribution of the coalescence time of two given paths, which we use in the proof of (B2) (see Theorems 3 and 4), and (b) as in [5], we resort to an FKG type of argument in an estimation in the proof of (B2), and for that we need to establish a monotonicity property of certain conditional probabilities on conditioning paths (see Proposition 1); this is also more delicate here than in [5].

Another drainage network for which the rescaled paths were shown to converge in distribution to the Brownian web is the one proposed by Scheidegger [12], which is equivalent to coalescing simple random walks. This case was treated in [8]. In the general context of drainage networks, our results may be seen as part of the scaling theory for those models. See [11, Section 2.5].

The GRS model may also be seen as coalescing random walks, in this case whose paths are noncrossing and have unbounded dependence among themselves. The other networks mentioned above may also be seen as noncrossing coalescing random walks. Coalescing nonsimple random walks which are independent before coalescence, and whose paths may thus cross one another, were studied in [3] and [10], and shown under suitable conditions to converge to the Brownian web when appropriately rescaled.

\section{Proof of Theorem 1}

This section is devoted entirely to the proof of Theorem 1. Let us fix a sequence $\delta=\delta_{n}=$ $1 / \sqrt{n}$ of positive numbers going to 0 as $n \rightarrow \infty$. We want to verify conditions (I1), (B1), and (B2) of Theorem 2 for $\mathcal{X}_{\delta_{n}}$. Due to the translation invariance of the model, (B1) follows from (I1) (as will be argued below). So we have only to verify (B2) and (I1). This will be done in Subsections 2.1 and 2.2-2.3, respectively. As a tool for both these verifications, we start by deriving a bound on the time of coalescence of two paths of the drainage model.

Coalescing random paths. Let $u, v \in \mathbb{Z}^{2}$ be such that $u(1) \leq v(1)$ and $u(2)=v(2)$. Consider $X^{u}$ and $X^{v}$, and, for $t \geq u(2)$, define

$$
Z_{t}=Z_{t}(u, v)=X^{v}(t)-X^{u}(t) .
$$

Note that $Z_{u(2)}=v(1)-u(1)$.

Remark 1. As argued in [9] (see Paragraph 3.1 in the proof of Theorem 2.1), $\left\{Z_{t}(u, v): t \geq\right.$ $u(2)\}$ is a nonnegative martingale in $\mathbb{L}^{2}$. Also, $Z_{t}(u, v) \rightarrow 0$ almost surely (a.s.) as $t \rightarrow \infty$.

Estimates for the tail of coalescence times. We need to control the tail of the meeting time of two coalescing random paths starting at the same time at a distance 1 apart. Define

$$
\tau:=\min \left\{t \geq 0: Z_{t}=0\right\}
$$

Theorem 3. There exists a constant $c_{2}>0$ such that $\mathrm{P}(\tau>t) \leq c_{2} / \sqrt{t}$.

In order to prove Theorem 3, it is enough to consider the case in which $u=(0,0)$ and $v=(1,0)$. By Remark 1, Skorokhod representation holds: that is to say, there exists a Brownian motion with coefficient of diffusion 1 starting from 1 and stopping times $0=T_{0}, T_{1}, T_{2}, \ldots$ satisfying

$$
Z_{t} \stackrel{\mathrm{D}}{=} B\left(T_{t}\right)
$$

where $0=T_{0}, T_{1}, T_{2}, \ldots$ are such that

$$
T_{t}=\inf \left\{s \geq T_{t-1}: B(s)-B\left(T_{t-1}\right) \notin\left(U_{t}\left(B\left(T_{t-1}\right)\right), V_{t}\left(B\left(T_{t-1}\right)\right)\right)\right\},
$$

where $\left\{\left(U_{t}(m), V_{t}(m)\right), t \geq 1, m \in \mathbb{Z}\right\}$ is a family of random independent vectors and, 
for all $m \in \mathbb{Z},\left(U_{t}(m), V_{t}(m)\right) \stackrel{\mathrm{D}}{=}\left(U_{t^{\prime}}(m), V_{t^{\prime}}(m)\right)$ and $\left(U_{t}(m), V_{t}(m)\right) \in\{(0,0), A\}$ with $A=\{\ldots,-2,-1\} \times\{1,2, \ldots\}$. Here $\stackrel{\text { D }}{=}$ denotes equality in distribution. Note that, since $\mathrm{P}\left(Z_{t} \geq 0\right)=1$, we have

$$
\mathrm{P}\left(U_{1}(m) \geq-m\right)=1 \quad \text { for all } m .
$$

The following result will be needed later on.

Lemma 1. For every $p<1$, there exists a constant $c_{1} \in(0,1)$ such that, for $m \geq 1$,

$$
\mathrm{P}\left(\left(U_{1}(m), V_{1}(m)\right)=(0,0)\right) \leq c_{1} .
$$

Proof. From the Skorokhod representation, we readily conclude that the left-hand side of (4) can be written as $\mathrm{P}\left(X_{1}^{(m, 0)}-X_{1}^{(0,0)}=m\right)$. By conditioning on $X^{(0,0)}$, a straightforward computation yields

$$
p^{2}+\frac{1-q^{2}}{2\left(1+q^{2}\right)} q^{2}
$$

as an upper bound for this probability for all $m$, where $q=1-p$, and this expression is strictly less than 1 for $p<1$.

Proof of Theorem 3. Let $\tau^{\prime}:=\min \{t \geq 0: B(t)=0\}$. From (2) and (3), we obtain

$$
\mathrm{P}(\tau>t)=\mathrm{P}\left(\tau^{\prime}>T_{t}\right)
$$

Now, for $\zeta>0$,

$$
\mathrm{P}\left(\tau^{\prime}>T_{t}\right) \leq \mathrm{P}\left(\tau^{\prime}>\zeta t\right)+\mathrm{P}\left(T_{t} \leq \zeta t\right) \leq \frac{c}{\sqrt{t}}+\mathrm{P}\left(T_{t} \leq \zeta t\right),
$$

where $c=c(\zeta) \in(0, \infty)$, and the second inequality follows by the well-known behavior of the tail of the distribution of $\tau^{\prime}$. By the Markov inequality we have

$$
\mathrm{P}\left(T_{t} \leq \zeta t\right)=\mathrm{P}\left(\mathrm{e}^{-\lambda T_{t}} \geq \mathrm{e}^{-\lambda \zeta t}\right) \leq \mathrm{e}^{\lambda \zeta t} \mathrm{E}\left(\mathrm{e}^{-\lambda T_{t}}\right) .
$$

We now write

$$
T_{t}=\sum_{i=1}^{t} S_{i}\left(Z_{i-1}\right),
$$

where $\left(S_{i}(k), i \geq 1, k \in \mathbb{Z}\right)$ are independent random variables. (Note that, for $k \in \mathbb{Z}$, ( $\left.S_{i}(k), i \geq 1\right)$ are not identically distributed.) Then, for $\lambda>0$,

$$
\begin{aligned}
\mathrm{E}\left(\mathrm{e}^{-\lambda T_{t}}\right) & =\mathrm{E}\left(\mathrm{E}\left(\exp \left\{-\lambda \sum_{i=1}^{t-1} S_{i}\left(Z_{i-1}\right)\right\} \exp \left\{-\lambda S_{t}\left(Z_{t-1}\right)\right\} \mid \mathcal{F}_{t-1}\right)\right) \\
& \leq \mathrm{E}\left(\exp \left\{-\lambda \sum_{i=1}^{t-1} S_{i}\left(Z_{i-1}\right)\right\}\right) \sup _{m \in \mathbb{Z}} \mathrm{E}\left(\mathrm{e}^{-\lambda S(m)}\right) \\
& \leq\left[\sup _{m \in \mathbb{Z}} \mathrm{E}\left(\mathrm{e}^{-\lambda S(m)}\right)\right]^{t},
\end{aligned}
$$

where $\mathcal{F}_{t}$ is the $\sigma$-algebra generated by $\left\{Z_{0}, Z_{1}, \ldots, Z_{t}\right\}$. Hence,

$$
\mathrm{P}\left(T_{t} \leq \zeta t\right) \leq\left[\mathrm{e}^{\lambda \zeta} \sup _{m \in \mathbb{Z}} \mathrm{E}\left(\mathrm{e}^{-\lambda S(m)}\right)\right]^{t},
$$


and, by Lemma 1 and the Skorokhod representation, we obtain (omitting $m \mathrm{~s}$ )

$$
\begin{aligned}
\mathrm{E}\left(\mathrm{e}^{-\lambda S}\right) \leq & \mathrm{P}((U, V)=(0,0)) \\
& +\mathrm{E}\left(\mathrm{e}^{-\lambda S} \mid(U, V)=(-1,1)\right)(1-\mathrm{P}((U, V)=(0,0))) \\
= & \left(1-\frac{1}{\cosh (\sqrt{2 \lambda})}\right) \mathrm{P}((U, V)=(0,0))+\frac{1}{\cosh (\sqrt{2 \lambda})} \\
\leq & \left(1-\frac{1}{\cosh (\sqrt{2 \lambda})}\right) c_{1}+\frac{1}{\cosh (\sqrt{2 \lambda})} .
\end{aligned}
$$

Indeed, we first note that $S$ is the hitting time of $(U, V)$ by $B$, and, thus, the case $(U, V)=$ $(-1,1)$ is dominated by the cases where $(U, V) \neq(0,0)$; this justifies the first inequality in (8). The equality is a well-known result (see, e.g. Theorem 5.7 of [4]), and the last inequality follows from Lemma 1. Since $c_{1}<1$ (uniformly in $m$ ), we may find $\lambda_{0}>0$ and $\zeta_{0}>0$ such that

$$
c_{3}:=\left(1-\frac{1}{\cosh \left(\sqrt{2 \lambda_{0}}\right)}\right) c_{1}+\frac{1}{\cosh \left(\sqrt{2 \lambda_{0}}\right)}<\mathrm{e}^{-\lambda_{0} \zeta_{0}} .
$$

Then, from (7),

$$
\mathrm{P}\left(T_{t}<\zeta_{0} t\right) \leq\left(c_{3} \mathrm{e}^{\lambda_{0} \zeta_{0}}\right)^{t}:=c_{4}^{t},
$$

where, from (9), $c_{4}$ may be taken to be less than 1 . It follows that there exists $c_{5} \in(0, \infty)$ such that $c_{4}^{t} \leq c_{5} / \sqrt{t}$. Now making $c_{2}=c+c_{5}$, the result follows from (6) and (10).

\subsection{Verification of condition (B2)}

The drainage network model is translation invariant, so we can eliminate $\sup _{\left(a, t_{0}\right) \in \mathbb{Z}^{2}}$ in (B2) and consider $a=t_{0}=0$.

To prove (B2), it suffices then to show that

$$
\varepsilon^{-1} \limsup _{N \rightarrow \infty} \mathrm{P}\left(\eta_{X}(0, t N, 0, \varepsilon \sqrt{N}) \geq 3\right) \rightarrow 0 \quad \text { if } \varepsilon \rightarrow 0+
$$

Define $n=\lfloor\varepsilon \sqrt{N}\rfloor$, and, for $j \in \mathbb{Z} \cap[0, n]$, let $X_{j}=\left\{\left(X^{(j, 0)}(k), k\right), k \in \mathbb{Z} \cap[0, t N]\right\}$ be the $\lfloor t N\rfloor$-step trajectory starting in $(j, 0)$. We next introduce the counting variable

$$
\eta^{\prime}=\left|\left\{X_{j}(t N): 0 \leq j \leq n\right\}\right|
$$

where $X_{j}(t N)=X^{(j, 0)}(\lfloor t N\rfloor)$. Then

$$
\mathrm{P}\left(\eta_{X}(0, t N ; 0, \varepsilon \sqrt{N}) \geq 3\right)=\mathrm{P}\left(\eta^{\prime} \geq 3\right) .
$$

Since

$$
\left\{\eta^{\prime} \geq 3\right\}=\bigcup_{j=1}^{n-1}\left\{X_{j-1}(t N)<X_{j}(t N)<X_{n}(t N)\right\}
$$


we have

$$
\begin{aligned}
\mathrm{P}\left(\eta^{\prime} \geq 3\right) \leq & \sum_{j=1}^{n-1} \mathrm{P}\left(X_{j-1}(t N)<X_{j}(t N)<X_{n}(t N)\right) \\
= & \sum_{j=1}^{n-1} \int_{\bar{\Pi}_{j}} \mathrm{P}\left(X_{j-1}(t N)<X_{j}(t N)<X_{n}(t N) \mid X_{j}=\pi\right) \mathrm{P}\left(X_{j}=\pi\right) \\
= & \sum_{j=1}^{n-1} \int_{\bar{\Pi}_{j}} \mathrm{P}\left(X_{j-1}(t N)<X_{j}(t N) \mid X_{j}=\pi\right) \\
& \quad \times \mathrm{P}\left(X_{j}(t N)<X_{n}(t N) \mid X_{j}=\pi\right) \mathrm{P}\left(X_{j}=\pi\right),
\end{aligned}
$$

where $\bar{\Pi}_{j}$ stands for the state space of $X_{j}$. The last equality follows from the fact that, given $X_{j}=\pi$, the events $\left\{X_{j-1}(t N)<X_{j}(t N)\right\}$ and $\left\{X_{j}(t N)<X_{n}(t N)\right\}$ are independent.

At this point we want to appeal to the Harris-FKG inequality. So we must establish monotonicity properties of the conditional probabilities on the right-hand side of (13).

We begin by introducing a partial order ' $\prec$ ' on $\bar{\Pi}_{j}$ as follows. Given $\pi_{1}$ and $\pi_{2} \in \bar{\Pi}_{j}$, we say that

$$
\pi_{1} \prec \pi_{2} \Longleftrightarrow \pi_{1}(\ell)-\pi_{1}(k) \leq \pi_{2}(\ell)-\pi_{2}(k)
$$

for every $\ell \geq k \geq 0, \ell, k \in \mathbb{Z} \cap[0, t N]$.

The partial order we have just introduced is an order on the increments of the trajectories belonging to events in $\bar{\Pi}_{j}$. Then, if the increments are increasing, the corresponding events will also be increasing.

Proposition 1. Let $\pi_{1}, \pi_{2} \in \bar{\Pi}_{j}$ be such that $\pi_{1} \prec \pi_{2}$. Then, for $k<j<n$,

$$
\begin{aligned}
& \mathrm{P}\left(X_{k}(t N)<X_{j}(t N) \mid X_{j}=\pi_{1}\right) \leq \mathrm{P}\left(X_{k}(t N)<X_{j}(t N) \mid X_{j}=\pi_{2}\right), \\
& \mathrm{P}\left(X_{j}(t N)<X_{n}(t N) \mid X_{j}=\pi_{1}\right) \geq \mathrm{P}\left(X_{j}(t N)<X_{n}(t N) \mid X_{j}=\pi_{2}\right) .
\end{aligned}
$$

Now, using Proposition 1 , and since the increments of $X_{j}$ are independent, we may apply the Harris-FKG inequality and find an upper bound for (13) as follows:

$$
\begin{aligned}
& \sum_{j=1}^{n-1} \int_{\bar{\Pi}_{j}} \mathrm{P}\left(X_{j-1}(t N)<X_{j}(t N) \mid X_{j}=\pi\right) \mathrm{P}\left(X_{j}=\pi\right) \\
& \quad \times \int_{\bar{\Pi}_{j}} \mathrm{P}\left(X_{j}(t N)<X_{n}(t N) \mid X_{j}=\pi\right) \mathrm{P}\left(X_{j}=\pi\right) \\
& =\sum_{j=1}^{n-1} \mathrm{P}\left(X_{j-1}(t N)<X_{j}(t N)\right) \mathrm{P}\left(X_{j}(t N)<X_{n}(t N)\right) \\
& \leq \sum_{j=1}^{n-1} \mathrm{P}\left(X_{j-1}(t N)<X_{j}(t N)\right) \mathrm{P}\left(X_{0}(t N)<X_{n}(t N)\right) \\
& \leq n \mathrm{P}\left(X_{0}(t N)<X_{1}(t N)\right) \mathrm{P}\left(X_{0}(t N)<X_{\varepsilon} \sqrt{N}(t N)\right) \\
& \leq \varepsilon \sqrt{N} \mathrm{P}(\tau>t N) \mathrm{P}\left(\tau_{\varepsilon, N}>t N\right),
\end{aligned}
$$

where $\tau$ is the coalescing time between the trajectories $X^{(0,0)}$ and $X^{(1,0)}$, and $\tau_{\varepsilon, N}$ is the time of coalescence of $X^{(0,0)}$ and $X^{(n, 0)}$. 
From (12) we obtain

$$
\varepsilon^{-1} \mathrm{P}\left(\eta_{\chi}(0, t N ; 0, \varepsilon \sqrt{N}) \geq 3\right) \leq \sqrt{N} \mathrm{P}(\tau>t N) \mathrm{P}\left(\tau_{\varepsilon, N}>t N\right) .
$$

It follows from Theorem 4 (in Subsection 2.2 below), in the case where $k=1, s_{0}=s_{1}=y_{0}=0$, and $y_{1}=\varepsilon$, that $\left(n^{-1 / 2} X^{(0,0)}(\lfloor n t\rfloor), n^{-1 / 2} X^{(n, 0)}(\lfloor n t\rfloor), t \geq 0\right)$ converges in distribution to a pair of coalescing Brownian motions. It follows that

$$
\limsup _{N \rightarrow \infty} \mathrm{P}\left(\tau_{\varepsilon, N}>t N\right)=\mathrm{P}\left(\tau_{\varepsilon, B}>t\right),
$$

where $\tau_{\varepsilon, B}$ is the time of coalescence of two independent Brownian motions with common diffusion coefficient $\sigma$ and starting at a distance $\varepsilon$ apart from each other at time zero. It is well known that $\mathrm{P}\left(\tau_{\varepsilon, B}>t\right)$ is $O(\varepsilon)$. On the other hand, by Lemma 3, there exists a constant $c_{2}^{\prime}>0$ such that $\mathrm{P}(\tau>t N)<c_{2}^{\prime} / \sqrt{t N}$. Taking lim $\sup _{N \rightarrow \infty}$ of both sides of (15) we obtain

$$
\varepsilon^{-1} \limsup _{n \rightarrow \infty} \mathrm{P}\left(\eta_{\chi}(0, t N ; 0, \varepsilon \sqrt{N}) \geq 3\right)=O(\varepsilon),
$$

which shows (11).

Proof of Proposition 1. It is enough to argue (14), since by the symmetry of the model we have

$$
\mathrm{P}\left(X_{j}(t N)<X_{n}(t N) \mid X_{j}=\pi\right)=\mathrm{P}\left(X_{2 j-n}(t N)<X_{j}(t N) \mid X_{j}=\pi^{-}\right),
$$

where $\pi^{-}$is the path of $\bar{\Pi}_{j}$ whose increments are the opposite of the respective ones of $\pi$.

The next reduction is that it is enough to consider $\pi_{1}$ and $\pi_{2}$ such that $\pi_{1}(s)=\pi_{2}(s)$ for $0 \leq s<t_{0}$ and $\pi_{2}(s)=\pi_{1}(s)+1$ for $s \geq t_{0}$ for some $t_{0} \in[0, t N] \cap \mathbb{Z}$. From now on, we will be dealing with such a pair of paths.

Remark 2. Given that $X_{j}=\pi$, the distribution of $(\Omega, \Upsilon)$ changes as follows. Let $I_{0}=$ $(j, 0)$, and, for $k \in[1, t N] \cap \mathbb{Z}$, let $I_{k}$ be the interval of integer numbers with endpoints $2 \pi(k-1)-\pi(k)$ and $\pi(k)$. ( $I_{k}$ can be a single point.) Then, outside $\bigcup_{k=0}^{\lfloor t N\rfloor} I_{k}$, the distribution of $(\Omega, \Upsilon)$ does not change. In $\bigcup_{k=0}^{\lfloor t N\rfloor} I_{k}$, the variables of $(\Omega, \Upsilon)$ are independent of the ones outside $\bigcup_{k=0}^{\lfloor t N\rfloor} I_{k}$, and they are also independent among themselves, except the pairs $(\omega(2 \pi(k-1)-\pi(k), k), v(\pi(k-1), k-1))$ for which $\left|I_{k}\right|>1$, whose distribution is described below. First, for each $k \in[1, t N] \cap \mathbb{Z}, \omega(\pi(k), k)=1$. If $\left|I_{k}\right|>1$ then $\omega\left(z_{1}, k\right)=0$ for $z_{1}$ in $I_{k}$ minus its endpoints, and $\omega(2 \pi(k-1)-\pi(k), k)$ is a Bernoulli random variable with parameter $p^{\prime}=p /(2-p)$. Given that $\omega(2 \pi(k-1)-\pi(k), k)=0$, then $v(\pi(k-1), k-1)$ is Bernoulli with parameter $\frac{1}{2}$; otherwise it is either 0 or 1 depending on whether $\pi(k)-\pi(k-1)$ is greater than or less than 0 .

A further point is that, given that $X_{j}=\pi$, the event $\left\{X_{k}(t N)<X_{j}(t N)\right\}$ depends on $\Omega$ only to the left of and including $\pi$, and on $\Upsilon$ only strictly to the left of $\pi$.

In order to prove (14), we couple the distributions of $(\Omega, \Upsilon)$ given $\pi_{1}$ and $\pi_{2}$ conveniently (in the relevant region of $\mathbb{Z}^{2}$ ). Let us denote by $\left(\omega_{i}(z), v_{i}(z)\right), z \in \mathbb{Z}^{2}$, the general term of $(\Omega, \Upsilon)$ given $\pi_{i}, i=1,2$, respectively. Then $\left(\omega_{2}(z), v_{2}(z)\right)=\left(\omega_{1}(z), v_{1}(z)\right)$ for $0 \leq z_{2}<t_{0}$ and $\left(\omega_{2}(z), v_{2}(z)\right)=\left(\omega_{1}\left(z_{1}-1, z_{2}\right), v_{1}\left(z_{1}-1, z_{2}\right)\right)$ for $0 \leq z_{2}>t_{0}$. The coupling on $z_{2}=t_{0}$ will be different in different cases. 
Case 1: $\pi_{1}\left(t_{0}\right)<\pi_{1}\left(t_{0}-1\right)$. In this case, we make

$$
\left(\omega_{2}\left(z_{1}, t_{0}\right), v_{2}\left(z_{1}, t_{0}\right)\right)=\left(\omega_{1}\left(z_{1}-1, t_{0}\right), v_{1}\left(z_{1}-1, t_{0}\right)\right) \quad \text { for } z_{1} \leq \pi_{2}\left(t_{0}\right)
$$

and

$$
\left(\omega_{2}\left(z_{1}, t_{0}\right), v_{2}\left(z_{1}, t_{0}\right)\right)=\left(\omega_{1}\left(z_{1}+1, t_{0}\right), v_{1}\left(z_{1}+1, t_{0}\right)\right) \text { for } z_{1}>\pi_{2}\left(t_{0}\right) .
$$

We readily check in this case that, for every realization $\left(\omega_{1}, v_{1}\right)(\cdot)$ of $(\Omega, \Upsilon)$ given $X_{j}=\pi_{1}$, $\left(\omega_{2}, v_{2}\right)(\cdot)$ given by the above coupling is a realization of $(\Omega, \Upsilon)$ given $X_{j}=\pi_{2}$, and that

$$
\left\{\left(\omega_{1}, v_{1}\right)(\cdot): X_{k}(t N)<X_{j}(t N)\right\} \subset\left\{\left(\omega_{2}, v_{2}\right)(\cdot): X_{k}(t N)<X_{j}(t N)\right\} .
$$

This then establishes (14) in this case.

Case 2: $\pi_{1}\left(t_{0}\right) \geq \pi_{1}\left(t_{0}-1\right)$. We start with a coupling which is the symmetric of the one above. It does not quite give us (16), but something nevertheless useful.

Auxiliary coupling. Given $\left(\omega_{2}, v_{2}\right)(\cdot)$, we make

$$
\left(\omega_{1}\left(z_{1}, t_{0}\right), v_{1}\left(z_{1}, t_{0}\right)\right)=\left(\omega_{2}\left(z_{1}+1, t_{0}\right), v_{2}\left(z_{1}+1, t_{0}\right)\right) \quad \text { for } z_{1} \geq \pi_{1}\left(t_{0}\right)
$$

and

$$
\left(\omega_{1}\left(z_{1}, t_{0}\right), v_{1}\left(z_{1}, t_{0}\right)\right)=\left(\omega_{2}\left(z_{1}-1, t_{0}\right), v_{2}\left(z_{1}-1, t_{0}\right)\right) \text { for } z_{1}<\pi_{1}\left(t_{0}\right) .
$$

We can readily check that this provides a coupling of $(\Omega, \Upsilon)$ given $X_{j}=\pi_{1}$ and $(\Omega, \Upsilon)$ given $X_{j}=\pi_{2}$. It is possible to find realizations of $\left(\omega_{2}, v_{2}\right)(\cdot)$ for which this coupling (14) does not hold. But the following can be readily checked:

$$
\left\{\left(\omega_{1}, v_{1}\right)(\cdot): X_{k}\left(t_{0}\right)<X_{j}\left(t_{0}\right)\right\} \subset\left\{\left(\omega_{2}, v_{2}\right)(\cdot): X_{k}\left(t_{0}\right)<X_{j}\left(t_{0}\right)\right\} .
$$

This will be used in the coupling discussed next.

We begin by pointing out that we could not find a direct coupling for which (16) holds, so we take an indirect route. We start by considering $D_{0}=X_{j}\left(t_{0}\right)-X_{k}\left(t_{0}\right)$. In order to insure the existence of a coupling of $\left(\omega_{1}, v_{1}\right)(\cdot)$ and $\left(\omega_{2}, v_{2}\right)(\cdot)$ satisfying $(16)$, it is enough to show that the distribution of $D_{0}$ given $X_{j}=\pi_{2}$ dominates that of $D_{0}$ given $X_{j}=\pi_{1}$. Indeed, if this holds then we can find a coupling of the two distributions such that the domination takes place almost surely. The respective random variables depend of course only on the history up to $t_{0}$, and, thus, we are free to choose any coupling of $\left(\omega_{1}, v_{1}\right)(\cdot)$ and $\left(\omega_{2}, v_{2}\right)(\cdot)$ above $t_{0}$, and choosing as in case 1 , we readily obtain (16).

In order to establish the abovementioned domination, we first define $\Delta=2\left(\pi_{1}\left(t_{0}\right)-\pi_{1}\left(t_{0}-\right.\right.$ 1)) and $\tilde{D}_{0}=\pi_{1}\left(t_{0}\right)-\Delta-X_{k}\left(t_{0}\right)$. Denote by $D_{i}, i=1,2$, the random variable whose distribution equals that of the conditional distribution of $\tilde{D}_{0}$ given that $X_{j}=\pi_{i}, i=1,2$, respectively. Then the abovementioned domination is equivalent to

$$
\mathrm{P}\left(D_{2} \geq k-1\right) \geq \mathrm{P}\left(D_{1} \geq k\right), \quad k \geq 0 .
$$

We will argue (18) using different couplings for different cases. The $k=0$ case of (18) is established as follows. If $\pi_{1}\left(t_{0}\right)=\pi_{1}\left(t_{0}-1\right)$, there is nothing to prove. If $\pi_{1}\left(t_{0}\right)>\pi_{1}\left(t_{0}-1\right)$ then $D_{1} \geq 0$ is equivalent to the left-hand side of (17), and $D_{2} \geq-1$ is equivalent to $D_{2} \geq 0$, which is in turn equivalent to the right-hand side of (17). So the auxiliary coupling can be used in this case. It can also be used if $k=1$. Indeed, in this case, $D_{2} \geq 0$ is equivalent to the right-hand side of (17), and $D_{1} \geq 1$ is either equivalent to the left-hand side of (17) (if $\left.\pi_{1}\left(t_{0}\right)=\pi_{1}\left(t_{0}-1\right)\right)$ or is in any case contained in it. 
For $k \geq 2$, we will resort to another coupling, described as follows.

1. For $z_{1}>\pi_{1}\left(t_{0}\right),\left(\omega_{2}\left(z_{1}, t_{0}\right), v_{2}\left(z_{1}, t_{0}\right)\right)=\left(\omega_{1}\left(z_{1}-1, t_{0}\right), v_{1}\left(z_{1}-1, t_{0}\right)\right)$.

2. For $\pi_{1}\left(t_{0}\right)-2\left(\pi_{1}\left(t_{0}\right)-\pi_{1}\left(t_{0}-1\right)\right) \leq z_{1} \leq \pi_{1}\left(t_{0}\right), \omega_{2}\left(z_{1}, t_{0}\right)=0$ and $v_{2}\left(z_{1}, t_{0}\right)$ are independent and identically distributed (i.i.d.) Bernoulli random variables with parameter $\frac{1}{2}$, independent of all else.

3. For $z_{1}=\pi_{1}\left(t_{0}\right)-2\left(\pi_{1}\left(t_{0}\right)-\pi_{1}\left(t_{0}-1\right)\right)-1,\left(\omega_{2}\left(z_{1}, t_{0}\right), v_{2}\left(z_{1}, t_{0}\right)\right)=\left(\xi \omega_{1}\left(z_{1}, t_{0}\right), \tilde{v}\right)$, where $\xi$ is a Bernoulli random variable with parameter $1 /(2-p)$, independent of all else, and $\tilde{v}$ depends only on $\xi \omega_{1}\left(z_{1}, t_{0}\right)$. If the latter random variable vanishes then $\tilde{v}$ is a Bernoulli random variable with parameter $\frac{1}{2}$, independent of all else; otherwise, $\tilde{v}=1$.

4. For $z_{1}<\pi_{1}\left(t_{0}\right)-2\left(\pi_{1}\left(t_{0}\right)-\pi_{1}\left(t_{0}-1\right)\right)-1$,

$$
\left(\omega_{2}\left(z_{1}, t_{0}\right), v_{2}\left(z_{1}, t_{0}\right)\right)=\left(\omega_{1}\left(z_{1}, t_{0}\right), v_{1}\left(z_{1}, t_{0}\right)\right)
$$

We readily check that, for every realization $\left(\omega_{1}, v_{1}\right)(\cdot)$ of $(\Omega, \Upsilon)$ given $X_{j}=\pi_{1},\left(\omega_{2}, v_{2}\right)(\cdot)$ given by the above coupling is a realization of $(\Omega, \Upsilon)$ given $X_{j}=\pi_{2}$, and that

$$
\left\{\left(\omega_{1}, v_{1}\right)(\cdot): D_{1} \geq k\right\} \subset\left\{\left(\omega_{2}, v_{2}\right)(\cdot): D_{2} \geq k\right\}
$$

in the remainder cases.

\subsection{Weak convergence to coalescing Brownian motions}

The main purpose of this and the next subsections is to prove condition (I1) of Theorem 2 .

Denote a single trajectory belonging to the drainage network model and starting from $a \in \mathbb{Z}$ at time $j \in \mathbb{Z}$ by $X^{a, j}=\left\{\left(X^{a, j}(l), l\right), l \geq j\right\}$. Then we have the following theorem.

Theorem 4. Let $\left(y_{0}, s_{0}\right),\left(y_{1}, s_{1}\right), \ldots,\left(y_{k}, s_{k}\right)$ be $k+1$ different points in $\mathbb{R}^{2}$ such that $s_{0} \leq$ $s_{1} \leq \cdots \leq s_{k}$, and if $s_{i-1}=s_{i}$ for some $i, i=1, \ldots, k$, then $y_{i-1}<y_{i}$. If $Z_{n}^{(i)}=\left\{Z_{n}^{(i)}(t):=\right.$ $\left.n^{-1 / 2} X_{n}^{(i)}(\lfloor n t\rfloor):=n^{-1 / 2} X^{\left\lfloor y_{i} \sqrt{n}\right\rfloor,\left\lfloor s_{i} n\right\rfloor}(\lfloor n t\rfloor), t \geq s_{i}\right\}$ then

$$
\left\{Z_{n}^{(i)}, i=0, \ldots, k\right\} \Rightarrow\left\{W^{(i)}, i=0, \ldots, k\right\}
$$

as $n \rightarrow \infty$, where the $W^{(i)}$ are $k+1$ coalescing Brownian motions with positive diffusion coefficient $\sigma$, starting at $\left\{\left(y_{0}, s_{0}\right), \ldots,\left(y_{k}, s_{k}\right)\right\}$.

Remark 3. Here, ' $\Rightarrow$ ' stands for weak convergence in $\Pi^{k+1}$ (the product of $k+1$ copies of $\Pi$, the path space (see [8])).

Remark 4. We denote by $\sigma^{2}$ the variance of $X^{0,0}(1)$, which we readily compute from its distribution, which is in turn straightforward to obtain, as $q\left(1+q^{2}\right) / p^{2}(1+q)^{2}$.

\subsection{Proof of Theorem 4}

The proof is divided into three parts. The arguments are somewhat standard, so we will be sketchy at a few points.

Part I. When $k=0$, the result follows from Donsker's invariance principle for single paths since $X^{a, j}$ is a random walk with variance $\sigma^{2}$. 
Part II. In this part we consider the case where $k \geq 1$ and $s_{0}=\cdots=s_{k}$ (and $y_{i-1}<y_{i}$ for every $i, i=1, \ldots, k)$. The general case will be treated in part III. Since the increments are stationary and spatially homogeneous, we will take $s_{0}=y_{0}=0$.

(a) We first treat the case in which $k=1$. Without loss of generality, we suppose that we are working in a probability space in which

$$
Z_{n}^{(1)} \stackrel{\text { a.s. }}{\longrightarrow} W^{(1)} \quad \text { as } n \rightarrow \infty .
$$

The following proposition, besides implying the result in the case in which $k=1$, is also the building block of the argument for the other cases of this part.

Proposition 2. The conditional distribution of $Z_{n}^{(0)}$ given $Z_{n}^{(1)}$ converges a.s. to the distribution of $W^{(0)}$ given $W^{(1)}$.

Proof. The strategy is to approximate the paths $Z_{n}^{(0)}$ and $Z_{n}^{(1)}$ before the time when they first come close (at a distance of order $n^{\alpha}, \alpha<\frac{1}{2}$ ) by independent paths, and then to show that after this time they meet quickly. Let us write

$$
X_{n}^{(i)}(l)=\left\lfloor y_{i} \sqrt{n}\right\rfloor+\sum_{h=1}^{l} S_{h}^{(i, n)},
$$

where, for each $i=0,1$ and $n \geq 1$, the $S_{h}^{(i, n)}, h \geq 1$, are i.i.d. random variables distributed as $X^{0,0}(1)$. Next, we introduce independent copies of $S_{h}^{(i, n)}, i=0,1$ and $n \geq 1$. For each $n \geq 1$, let $\left\{\tilde{S}_{h}^{(i, n)}, h \geq 1, i=0,1\right\}$ be i.i.d. random variables such that $\tilde{S}_{1}^{(0, n)} \sim X^{0,0}(1)$ and define random variables $\hat{S}_{h}^{(i, n)}$ by

$$
\hat{S}_{h}^{(i, n)}= \begin{cases}S_{h}^{(i, n)} & \text { if }\left|S_{h}^{(i, n)}\right| \leq n^{\alpha}, \\ \tilde{S}_{h}^{(i, n)} & \text { otherwise, }\end{cases}
$$

where $\alpha$ is a positive number to be specified later. Moreover, for $l \geq 1$, let $\hat{X}_{n}^{(i)}(l)=\left\lfloor y_{i} \sqrt{n}\right\rfloor+$ $\sum_{h=1}^{l} \hat{S}_{h}^{(i, n)}$. Define

$$
\hat{\tau}_{n}=\min \left\{l \geq 1: \hat{X}_{n}^{(1)}(l)-\hat{X}_{n}^{(0)}(l) \leq 3 n^{\alpha}\right\}
$$

and

$$
\tau_{n}=\min \left\{l \geq 1: X_{n}^{(1)}(l)-X_{n}^{(0)}(l) \leq 3 n^{\alpha}\right\},
$$

and let

$$
\tilde{X}_{n}^{(i)}(l)= \begin{cases}\hat{X}_{n}^{(i)}(l) & \text { if } l \leq \hat{\tau}_{n}, \\ \hat{X}_{n}^{(i)}\left(\hat{\tau}_{n}\right)+\sum_{h>\hat{\tau}_{n}} \check{S}_{h}^{(i, n)} & \text { otherwise, }\end{cases}
$$

where

$$
\check{S}_{h}^{(i, n)}= \begin{cases}\tilde{S}_{h}^{(0, n)} & \text { if } i=0, \\ S_{h}^{(1, n)} & \text { if } i=1 .\end{cases}
$$

From the exponential tail of the distribution of $S_{h}^{(i, n)}$ (which is independent of $i$ ) we readily obtain the following result.

Lemma 2. Given $\beta<\infty$, the complement of the event

$$
A_{n}:=\left\{S_{h}^{(i, n)}=\hat{S}_{h}^{(i, n)}, i=1, \ldots, n^{\beta} ; i=0,1\right\}
$$

has probability going to 0 super-polynomially fast as $n \rightarrow \infty$. 
Corollary 1. The event $B_{n}:=\left\{X_{n}^{(i)}(l)=\hat{X}_{n}^{(i)}(l), l=1, \ldots, n^{\beta} ; i=0,1\right\}$ is such that $\mathrm{P}\left(B_{n}^{\mathrm{c}}\right) \rightarrow 0$ super-polynomially fast as $n \rightarrow \infty$.

From the construction, the properties stated in the following result are readily verified.

Lemma 3. For each $n \geq 1, \tilde{X}_{n}^{(0)}:=\left\{\tilde{X}_{n}^{(0)}(l), l \geq 1\right\}$ and $\tilde{X}_{n}^{(1)}:=\left\{\tilde{X}_{n}^{(1)}(l), l \geq 1\right\}$ are independent processes and $\bar{X}_{n}^{(i)} \sim \tilde{X}_{n}^{(i)}$ for each fixed $\bar{i}=0,1$.

Corollary 2. Let $\tilde{Z}_{n}^{(i)}(t):=n^{-1 / 2} \tilde{X}_{n}^{(i)}(\lfloor n t\rfloor), t \geq 0$. Then,

1. $\tilde{Z}_{n}^{(1)} \rightarrow W^{(1)}$ a.s. as $n \rightarrow \infty$;

2. $\left(\tilde{Z}_{n}^{(0)}, \tilde{Z}_{n}^{(1)}\right) \Rightarrow\left(\tilde{W}^{(0)}, W^{(1)}\right)$, where $\tilde{W}^{(0)} \sim W^{(0)}$, and $\tilde{W}^{(0)}$ and $W^{(1)}$ are independent;

3. the conditional distribution of $\left\{\tilde{Z}_{n}^{(0)}(t), t \leq \hat{\tau}_{n} / n\right\}$ given $\tilde{Z}_{n}^{(1)}$ converges a.s. to that of $\left\{\tilde{W}^{(0)}(t), t \leq \tau\right\}$ given $W^{(1)}$, where $\tau=\inf \left\{t \geq 0: \tilde{W}^{(0)}(t)=W^{(1)}(t)\right\}$.

Corollary 3. The conditional distribution of $\left\{Z_{n}^{(0)}(l), l \leq \tau_{n}\right\}$ given $Z_{n}^{(1)}$ converges a.s. to that of $\left\{\tilde{W}^{(0)}(t), t \leq \tau\right\}$ given $W^{(1)}$.

Lemma 4. For $\alpha<\frac{1}{2}$, there exists $\gamma=\gamma(\alpha)<1$ such that, as $n \rightarrow \infty$,

$$
\mathrm{P}\left(X_{n}^{(0)}(l) \neq X_{n}^{(1)}(l), l=\tau_{n}, \ldots, \tau_{n}+n^{\gamma}\right) \rightarrow 0 .
$$

Corollary 4. The process $\left\{Z_{n}^{(0)}(t), t \geq \tau_{n} / n\right\}$ converges in probability to $\left\{W^{(1)}(t), t \geq \tau\right\}$.

Then, Proposition 2 follows directly from Corollaries 3 and 4, and the Markov property.

Proof of Corollary 2. 1. Let $\beta>\frac{7}{2}$ be fixed. We claim that there exists a finite constant $C$ and $\theta>1$ such that

$$
\mathrm{P}\left(\hat{\tau}_{n}>n^{\beta}\right) \leq \frac{C}{n^{\theta}} .
$$

To argue this, we first note that $Y^{(n)}:=\hat{X}_{n}^{(1)}-\hat{X}_{n}^{(0)}$ is a random walk on the integers starting from $\left\lfloor y_{i} \sqrt{n}\right\rfloor$ and with increments distributed as $Y^{(n)}(1)=\hat{S}_{1}^{(1, n)}-\hat{S}_{1}^{(0, n)}$. We may then readily note that $\hat{\tau}_{n}$ is dominated by the sum of $\left\lfloor y_{i} \sqrt{n}\right\rfloor$ copies of $\tilde{\tau}_{n}$, the hitting time of the negative integers by a random walk with increments distributed as $Y^{(n)}(1)$, started at the origin. We then have

$$
\mathrm{P}\left(\hat{\tau}_{n}>n^{\beta}\right) \leq c_{1} \sqrt{n} \mathrm{P}\left(\tilde{\tau}_{n}>c_{2} n^{\beta-1 / 2}\right) \leq \frac{C}{n^{(\beta-3 / 2) / 2}},
$$

where the first bound is straightforward from the above domination, and the last bound follows from a standard fact on the tail of the distribution of hitting times for one-dimensional random walks with finite second moment, like $Y^{(n)}$. The claim is thus justified.

We can then use (19), Corollary 1, and (20), together with the Borel-Cantelli lemma, to conclude.

2. The proof of this part is immediate from Lemma 4 and Donsker's theorem.

3. We take a version of $\tilde{Z}_{n}^{(0)}$, say $\bar{Z}_{n}^{(0)}$, such that

$$
\left(\bar{Z}_{n}^{(0)}, \tilde{Z}_{n}^{(1)}\right) \stackrel{\text { a.s. }}{\longrightarrow}\left(\bar{W}^{(0)}, W^{(1)}\right) \quad \text { as } n \rightarrow \infty,
$$

with $\left(\bar{W}^{(0)}, W^{(1)}\right) \sim\left(\tilde{W}^{(0)}, W^{(1)}\right)$, and claim that $\bar{\tau}_{n} \rightarrow \bar{\tau}$ a.s. as $n \rightarrow \infty$, where $\bar{\tau}_{n}=\min \{t \geq$ 0: $\left.\tilde{Z}_{n}^{(1)}(t)-\tilde{Z}_{n}^{(1)}(t) \leq 3 n^{\alpha-1 / 2}\right\}$ and $\bar{\tau}=\inf \left\{t \geq 0: \bar{W}^{(0)}(t)=W^{(1)}(t)\right\}$. Indeed, the almostsure convergence of $\bar{\tau}_{n}$ follows from the property that, with probability 1, two independent Brownian trajectories will instantaneously cross after touching, i.e.

$$
\mathrm{P}\left(\text { for all } \varepsilon>0, \bar{W}^{(0)}(t)>W^{(1)}(t) \text { for some } t \leq \bar{\tau}+\varepsilon\right)=1,
$$


and the following deterministic result (whose proof is an exercise). The convergence in distribution in the original space follows. This completes the proof.

Lemma 5. Let $f_{n}, g_{n}, f, g: \mathbb{R}^{+} \rightarrow \mathbb{R}$ be continuous functions such that $f(0)<g(0)$, and let $T=\inf \{t \geq 0: f(t)=g(t)\}$ be finite and have the property that, for every $\delta>0$, there exists $t \in[T, T+\delta]$ with $f(t)>g(t)$. Suppose also that $\lim _{n \rightarrow \infty} \sup _{0 \leq t \leq T+1}\left|f_{n}(t)-f(t)\right|=0$ and that $\lim _{n \rightarrow \infty} \sup _{0 \leq t \leq T+1}\left|g_{n}(t)-g(t)\right|=0$, and let $T_{n}=\inf \left\{t \geq 0: g_{n}(t)-f_{n}(t) \leq \varepsilon_{n}\right\}$, where $\left(\varepsilon_{n}\right)$ is a given sequence of numbers vanishing as $n \rightarrow \infty$. Then, $\lim _{n \rightarrow \infty} T_{n}=T$.

Proof of Corollary 3. The proof is immediate from Corollary 1 and part 3 of Corollary 2.

Proof of Lemma 4. Let $\sigma_{n}=\inf \left\{l \geq \tau_{n}: X_{n}^{(0)}(l)=X_{n}^{(1)}(l)\right\}-\tau_{n}$. It can be readily checked that $\sigma_{n}$ is dominated by $\tilde{\sigma}_{n}$, the hitting time of 0 by $Z_{t}\left((0,0),\left(\left\lfloor 3 n^{\alpha}\right\rfloor, 0\right)\right)$ (see (1)). Proceeding as in the proof of Theorem 3, we find that

$$
\mathrm{P}\left(\tilde{\sigma}_{n}>n^{\gamma}\right)=\mathrm{P}\left(\sigma_{n}^{\prime}>T_{n}\right) \leq \mathrm{P}\left(\sigma_{n}^{\prime}>\zeta n^{\gamma}\right)+\mathrm{P}\left(T_{n^{\gamma}} \leq \zeta n^{\gamma}\right),
$$

where $\sigma_{n}^{\prime}$ is the hitting time of 0 by a Brownian motion started at $\left\lfloor 3 n^{\alpha}\right\rfloor$ (see (5) and (6)). Now choosing $\gamma$ in $(2 \alpha, 1)$, we readily check that the first term on the right-hand side of (21) vanishes as $n \rightarrow \infty$. The second term on the right-hand side of (21) also vanishes as $n \rightarrow \infty$ by (10), and the proof is complete.

Proof of Corollary 4. By (19) and Lemma 4, it is enough to show that, for every $\varepsilon>0$, as $n \rightarrow 0$,

$$
\mathrm{P}\left(\max _{0 \leq l \leq n}\left(X_{n}^{(1)}\left(l+\tau_{n}\right)-X_{n}^{(0)}\left(l+\tau_{n}\right)\right)>\varepsilon \sqrt{n}\right) \rightarrow 0 .
$$

By the Markov property and an elementary domination argument, the left-hand side of (22) is bounded above by

$$
\mathrm{P}\left(\max _{0 \leq l \leq n^{\gamma}} \hat{Z}_{l}^{(n)}>\varepsilon \sqrt{n}\right),
$$

where $\hat{Z}_{l}^{(n)}=Z_{l}\left((0,0),\left(\left\lfloor 3 n^{\alpha}\right\rfloor, 0\right)\right)$ (see (1)). Now, by Doob's inequality, (23) is bounded above by a constant times $\mathrm{E}\left(\hat{Z}_{n^{\gamma}}^{(n)}\right) / \sqrt{n} \leq 3 n^{\alpha-1 / 2} \rightarrow 0$ as $n \rightarrow \infty$. This completes the proof.

(b) We now consider the case in which $k>1$. It suffices to prove that

$$
\mathrm{E}\left(f_{0}\left(Z_{n}^{(0)}\right) \cdots f_{k}\left(Z_{n}^{(k)}\right)\right) \rightarrow \mathrm{E}\left(f_{0}\left(W^{(0)}\right) \cdots f_{k}\left(W^{(k)}\right)\right) \quad \text { as } n \rightarrow \infty
$$

for any $f_{0}, \ldots, f_{k} \in C_{b}(\Pi, \mathbb{R})$, the space of all real-valued and bounded continuous functions defined on $\Pi$.

The left-hand side of (24) is equivalent to

$$
\mathrm{E}\left(f_{1}\left(Z_{n}^{(1)}\right) \mathrm{E}\left(f_{0}\left(Z_{n}^{(0)}\right) f_{2}\left(Z_{n}^{(2)}\right) \cdots f_{k}\left(Z_{n}^{(k)}\right) \mid Z_{n}^{(1)}\right)\right) .
$$

It follows from Lemma 6, below, that the conditional expectation equals

$$
\mathrm{E}\left(f_{0}\left(Z_{n}^{(0)}\right) \mid Z_{n}^{(1)}\right) \mathrm{E}\left(f_{2}\left(Z_{n}^{(2)}\right) \cdots f_{k}\left(Z_{n}^{(k)}\right) \mid Z_{n}^{(1)}\right) .
$$

Since $Z_{n}^{(1)} \stackrel{\text { a.s. }}{\longrightarrow} W^{(1)}$, by the inductive hypothesis, the conditional expectations above converge, a.s., to $\mathrm{E}\left(f_{0}\left(W^{(0)}\right) \mid W^{(1)}\right)$ and $\mathrm{E}\left(f_{2}\left(W^{(2)}\right) \cdots f_{k}\left(W^{(k)}\right) \mid W^{(1)}\right)$, respectively. On the other hand, by the dominated convergence theorem we obtain the convergence of (25) to

$$
\mathrm{E}\left(f_{1}\left(W^{(1)}\right) \mathrm{E}\left(f_{0}\left(W^{(0)}\right) \mid W^{(1)}\right) \mathrm{E}\left(f_{2}\left(W^{(2)}\right) \cdots f_{k}\left(W^{(k)}\right) \mid W^{(1)}\right)\right),
$$


which equals

$$
\mathrm{E}\left(f_{1}\left(W^{(1)}\right) \mathrm{E}\left(f_{0}\left(W^{(0)}\right) f_{2}\left(W^{(2)}\right) \cdots f_{k}\left(W^{(k)}\right) \mid W^{(1)}\right)\right) .
$$

Since (26) equals the right-hand side of (24), we have the result.

Lemma 6. For each $n \geq 1$, given $Z_{n}^{(1)}, Z_{n}^{(0)}$ is independent of $\left(Z_{n}^{(2)}, \ldots, Z_{n}^{(k)}\right)$.

Proof. The process $Z_{n}^{(0)}$ depends only on the variables of $(\Omega, \Upsilon)$ to the left of and including $Z_{n}^{(1)}$, and $\left(Z_{n}^{(2)}, \ldots, Z_{n}^{(k)}\right)$ depends only on the variables of $(\Omega, \Upsilon)$ to the right of and including $Z_{n}^{(1)}$. The result follows from the product structure of the conditional distribution of $(\Omega, \Upsilon)$ given $Z_{n}^{(1)}$ (see Remark 2), and the facts that, given $Z_{n}^{(1)}$, the variables of $\Omega$ along $Z_{n}^{(1)}$ are fixed, and when $Z_{n}^{(0)}$ depends on the variable of $\Upsilon$ in a particular position of $Z_{n}^{(1)}$ (this happens when, and only when, the increment of $Z_{n}^{(1)}$ from the given position is strictly positive), then $\left(Z_{n}^{(2)}, \ldots, Z_{n}^{(k)}\right)$ does not depend on that particular variable, and vice-versa. This completes the proof.

Part III. We now take on the general case and proceed by induction on $k \geq 0$. The case in which $k=0$ was argued in part I above. Suppose now that, for some $k \geq 1$, the result holds up to $k-1$, and let $\left(y_{0}, s_{0}\right), \ldots,\left(y_{k}, s_{k}\right)$ be as in the statement of Theorem 4 . If the condition on $\left(s_{i}\right)$ of part II holds then there is nothing to prove. Otherwise, there exists $i \in\{1, \ldots, k\}$ such that $s_{i}>s_{i-1}$. Let $i_{0}$ be the maximum between such $i$ s. By the induction hypothesis,

$$
\left(\left\{Z_{n}^{(i)}(t), s_{i} \leq t \leq s_{i_{0}}\right\}, 0 \leq i<i_{0}\right) \Rightarrow\left(\left\{W^{(i)}(t), s_{i} \leq t \leq s_{i_{0}}\right\}, 0 \leq i<i_{0}\right),
$$

and we can suppose that such convergence occurs a.s. By the Markov property and part II (strengthened to accommodate the case where the $y_{i}$ s may depend on $n$ and converge to, say, $\tilde{y}_{i}$ as $n \rightarrow \infty$; this requires minor changes in the argument above), we have, given (27),

$$
\left(\left\{Z_{n}^{(i)}(t), t \geq s_{i_{0}}\right\}, 0 \leq i \leq k+1\right) \Rightarrow\left(\left\{W^{(i)}(t), t \geq s_{i_{0}}\right\}, 0 \leq i \leq k+1\right) .
$$

Then, Theorem 4 follows from (27), (28), and the Markov property.

\subsection{Verification of (B1)}

In this subsection we prove condition (B1), which is in fact a consequence of condition (I1) for this model. We note that $\eta_{X_{n}}(0, t ; 0, \varepsilon) \geq 2$ if and only if the two trajectories starting at time 0 from the border of the interval $[0, \varepsilon]$ have not met up to time $t$. By Theorem 4 , the rescaled trajectories of a finite collection of paths belonging to the drainage network model converge to those of coalescing Brownian motion. Then, it is straightforward to find, for the drainage network model, that

$$
\limsup _{n \rightarrow \infty} \mathrm{P}\left(\eta_{x_{n}}(0, t ; 0, \varepsilon) \geq 2\right)=2 \Phi\left(\frac{\varepsilon}{\sqrt{2 t}}\right)-1,
$$

where $\Phi(\cdot)$ is the standard normal distribution function. This together with the translation invariance of the model concludes the verification of (B1).

\section{Acknowledgements}

This work started as part of the master's project of the third author and was completed during the postdoc project of the first author, both at IME-USP and financed by FAPESP. We thank Pablo Ferrari for discussions on the coupling argument of Subsection 2.1. We also thank an anonymous referee, whose suggestions helped to improve the presentation. 


\section{References}

[1] Arratia, R. (1981). Coalescing Brownian motions and the voter model on $\mathbb{Z}$. Unpublished manuscript.

[2] Arratia, R. (1981). Limiting point processes for rescalings of coalescing and annihilating random walks on $\mathbb{Z}^{d}$. Ann. Prob. 9, 909-936.

[3] Belhaouari, S., Mountford, T., Sun, R. and Valle, G. (2006). Convergence results and sharp estimates for the voter model interfaces. Electron. J. Prob. 11, 768-801.

[4] Durrett, R. (1996). Probability: Theory and Examples, 2nd edn. Duxbury Press, Belmont, CA.

[5] Ferrari, P. A., Fontes, L. R. G. And Wu, X.-Y. (2005). Two-dimensional Poisson trees converge to the Brownian web. Ann. Inst. H. Poincaré Prob. Statist. 41, 851-858.

[6] Ferrari, P. A., Landim, C. and Thorisson, H. (2004). Poisson trees, succession lines and coalescing random walks. Ann. Inst. H. Poincaré Prob. Statist. 40, 141-152.

[7] Fontes, L. R. G., Isopi, M., Newman, C. M. and Ravishankar, K. (2002). The Brownian web. Proc. Nat. Acad. Sci. USA 99, 15888-15893.

[8] Fontes, L. R. G., Isopi, M., Newman, C. M. and Ravishankar, K. (2004). The Brownian web: characterization and convergence. Ann. Prob. 32, 2857-2883.

[9] Gangopadhyay, S., Roy, R. And Sarkar, A. (2004). Random oriented trees: a model of drainage networks. Ann. App. Prob. 14, 1242-1266.

[10] Newman, C. M., Ravishankar, K. And Sun, R. (2005). Convergence of coalescing nonsimple random walks to the Brownian web. Electron. J. Prob. 10, 21-60.

[11] Rodriguez-Iturbe, I. And Rinaldo, A. (1997). Fractal River Basins: Chance and Self-Organization. Cambridge University Press.

[12] Scheidegger, A. E. (1967). A stochastic model for drainage patterns into an intramontane trench. Bull. Ass. Sci. Hydrol. 12, 15-20.

[13] Tóth, B. And Werner, W. (1998). The true self-repelling motion. Prob. Theory Relat. Fields 111, $375-452$. 